\title{
Preliminary Study of the GSTM1 Null Polymorphism and History of Tobacco Smoking among Oral Cancer Patients in Northeastern Thailand
}

\author{
Sitakan Natphopsuk ${ }^{1,2}$, Wannapa Settheetham-Ishida ${ }^{1 *}$, Sophida Phuthong ${ }^{1}$, \\ Takafumi Ishida ${ }^{3}$
}

\begin{abstract}
Risks with GSTM1 genotypes and potential roles of smoking in the susceptibility to oral squamous cell carcinoma (OSCC) were studied in Northeastern Thailand. Study subjects were 79 histologically-confirmed OSCC cases ( 31 men, 48 women) and 79 age- and sex-matched healthy controls ranging in age from 25 to 84 years. GSTM1 genotyping was achieved by two independent PCR assays. The GSTM1 null allele and the homozygous genotype did not increase risk of $\mathrm{OSCC}$ vs the wild type allele and the remaining genotypes. When the focus was on the smoking habit, male subjects who smoked $\geq 10$ or $\geq 35$ years were at significantly increased risk for OSCC with adjusted ORs of 4.88 [95\% CI, 1.41-16.87, $\mathrm{p}=0.012]$ or 4.94 [95\% CI, 1.62-15.12, $\mathrm{p}=\mathbf{0 . 0 0 5}$ ], respectively. A higher risk for OSCC was found for smoking amount; those who smoked $>5$ or $>10$ pack-years were at a higher risk with adjusted OR of 4.46 [95\% CI; 1.45-13.74, $\mathrm{p}=0.009$ ] or 3.89 [95\% CI; 1.34-11.28, $\mathrm{p}=0.012$ ], respectively. There are certain smoking patterns that give greater risks and thus both smoking duration and pack-years should be taken into consideration in tobacco related cancer prevention.
\end{abstract}

Keywords: Oral cancer - GSTM1 - tobacco smoking - Northeast Thailand

Asian Pac J Cancer Prev, 17 (2), 739-742

\section{Introduction}

Oral cancer is a serious public health problem in many countries due to its low survival rate and the resultant decreased quality of life (Rogers et al., 2009; Nagler et al., 2010; Larizadeh and Shabani, 2012). In Thailand, the incidence rate (per 100,000) is 6.2 among females and 3.9 among males (Vatanasapt et al., 2011). Exposure to environmental carcinogens-such as betel quid chewing, tobacco smoking and alcohol drinking-has been identified as a common cause of the cancer (Rodriguez et al., 2004; Petti, 2009; Loyha et al., 2012). Tobacco smoke contains several carcinogens such as nitrosamines, polycyclic aromatic hydrocarbons (PAH), benzo[a]pyrene and aromatic amines (Shields, 2002; Mukherjee and Kumar, 2010). These lipophilic carcinogens are metabolized by phase I and phase II enzymes (Hayes and Pulford, 1995; Sreelekha et al., 2001); therefore, both environmental and host susceptibility factors may be relevant to the etiology of this nonhereditary cancer.

Glutathione S-transferase $\mathrm{Mu} 1$ (GSTM1) is one of the human glutathione S-transferases belonging to phase II detoxification enzymes. It plays a key role in the detoxification of the carcinogenic electrophiles of aflatoxin and PAHs in tobacco smoke (Nair et al., 1999;
Hahn et al., 2002; Drummond et al., 2004). The absence of the homozygous allele of the GSTM1 gene (GSTM1 null genotype) results in a complete loss of enzyme activity for binding with genotoxic substrates; including the epoxides derived from aflatoxin and PAHs, resulting in a decreased capacity to detoxify carcinogens (Hayes and Pulford, 1995, Sreelekha et al., 2001). It is believed that individuals with the GSTM1 null genotype lack the functional ability to detoxify the ultimate form of carcinogens and are, therefore, susceptible to cancer in various organs (including the lung, bladder, oral and nasopharynx) (Schnakenberg et al., 2000; Shield PG., 2002; Tiwawech et al., 2005; Liu et al., 2014; Zakiullah et al., 2015). Results of many other studies dealing with the association between GSTM1 polymorphism in some of these cancers have, however, proven contradictory (Tanimoto et al., 1999; Hahn et al., 2002; Natphopsuk et al., 2015). Geographical and ethnic differences as well as the method of genotypic detection may be responsible for the apparently conflicting data. The real risk for all GSTM1 genotypes for oral cancer can be identified by distinguishing the homozygous wild-type from heterozygous individuals. In the current study, we investigated whether the GSTMI genotype was associated with risk of oral cancer and evaluate the potential role of smoke in the susceptibility to oral cancer in Northeastern Thailand.

${ }^{1}$ Department of Physiology, Khon Kaen University, Khon Kaen, ${ }^{2}$ Chulabhorn International College of Medicine, Thammasat University, Thailand, ${ }^{3}$ Department of Biological Sciences, Graduate School of Science, The University of Tokyo, Tokyo, Japan *For correspondence: wannapa@kku.ac.th 


\section{Materials and Methods}

\section{Study population}

Study subjects comprised 79 cases (31 men, 48 women) and 79 age- and sex-matched healthy controls ranging in age from 25 to 84 years. These subjects attended Khon Kaen General Hospital, Srinagarind (Khon Kaen University) Hospital, Khon Kaen Regional Hospital or Sappasitthiprasong Ubon Ratchathani Hospital, all in Northeast Thailand between July 2010 and April 2011. Cases were histologically-confirmed as oral squamous cell carcinoma (OSCC), while controls were selected from among general walk-in patients at the Out-Patient Department of the hospitals and who were diagnosed with non-cancer conditions. Informed consent and a thorough interview were taken before blood collection. We only interviewed patients who consented to participate in the study: we used a standardized questionnaire concerning their tobacco use. The life-time smoking consumption history included the smoking status (smoke or never), smoking duration (year) and amount of smoking used (pack-year) were collected. The multiplying of number of packs of cigarettes smoked per day by the number of years the person has smoked was estimated as a smoking pack-year (one pack contains 20 cigarettes). Our study was reviewed and approved by the Ethics Committee of Khon Kaen University (HE 521344).

\section{Detection of GSTM1 polymorphism}

Blood (3 ml) from each subject was collected in an EDTA tube and genomic DNAs were extracted by using the GF-1 Blood DNA Extraction Kit (Vivantis, USA).
GSTM1 genotype was determined by the two independent PCR assays described by Natphopsuk et al. (2015). To identify the GSTM1 null allele, a short-PCR amplification (Tiwawech et al., 2005) was performed using a primer pairs (5'-GAA CTC CCT GAA AAG CTA AAG C-3' and 5'-GTT GGG CTC AAA TAT ACG GTG G-3'); an amplification of the $\beta$-globin was used as an internal control. PCR products were analyzed by $2.5 \%$ agarose gel electrophoresis. To identify the GSTMI heterozygous allele, a long-PCR amplification was performed (Roodi et al., 2004) using a primer set of M3 (5'-CCT GTT GAA GGA GCT TAT GCT GAA-3') and M4 (5'- TTC TGA GGA CTG GAC TGA TGA TC-3') with KOD FX (Toyobo, Japan). The PCR product (14kb) was analyzed by $0.5 \%$ agarose gel electrophoresis.

\section{Statistical analyses}

The Chi-square test was used to compare genotypic frequencies between the case and control. The OR and 95\% CI were calculated to determine the association between selected variables and the risk for OSSC between the GSTM1 genotypes in both cases and controls. Statistical analyses were conducted using the statistical software 800-STATA for PC. A $P$-value of $<0.05$ was required for statistical significance.

\section{Results}

The respective overall frequency of the GSTM1 null genotype among the controls and cases was $64.6 \%$ and $54.4 \%$, respectively. The genotype distribution was in Hardy-Weinberg equilibrium. The respective genotype

Table 1. GSTM1 Polymorphism and Oral Cancer

\begin{tabular}{|c|c|c|c|c|}
\hline Variables & Cases n $(\%)$ & Controls n $(\%)$ & OR [95\% CI, p-value] & Adjusted OR ${ }^{\mathrm{a}}[95 \% \mathrm{CI}, \mathrm{p}$-value $]$ \\
\hline \multicolumn{5}{|c|}{ GSTM1 polymorphism } \\
\hline$+/+$ and $+/-$ & $36(45.57)$ & $28(35.44)$ & 1 & 1 \\
\hline$-1-$ & $43(54.43)$ & $51(64.56)$ & $0.66[0.33-1.30,0.198]$ & $0.62[0.32-1.21,0.161]$ \\
\hline$+/+$ & $6(7.59)$ & $7(8.86)$ & 1 & 1 \\
\hline$+/-$ & $30(37.97)$ & $21(26.58)$ & $1.67[0.41-6.91,0.411]$ & $2.07[0.58-7.40,0.264]$ \\
\hline$-/-$ & $43(54.43)$ & $51(64.56)$ & $0.98[0.26-3.83,0.978]$ & $1.11[0.33-3.73,0.865]$ \\
\hline
\end{tabular}

aadjusted multiple logistic regression

Table 2. GSTM1 Polymorphism and Oral Cancer in Non-smoking Females

\begin{tabular}{lcccc}
\hline GSTM1 polymorphism & Cases n (\%) & Controls n (\%) & OR [95\% CI, p-value] & Adjusted OR ${ }^{\text {a }}$ [95\% CI, p-value] \\
\hline$+/+$ & $2(4.44)$ & $4(8.33)$ & 1 & 1 \\
$+/-$ & $20(44.4)$ & $14(29.17)$ & $2.86[0.34-34.78,0.381]$ & $2.86[0.46-17.80,0.261]$ \\
$-/-$ & $23(51.11)$ & $30(62.5)$ & $1.53[0.20-18.21,1.000]$ & $1.53[0.26-9.11,0.638]$ \\
\hline
\end{tabular}

adjusted multiple logistic regression

Table 3. Smoking Status and Risk for Oral Cancer in Males

\begin{tabular}{lrccc}
\hline Duration (year) & Cases n $(\%)$ & Controls n $(\%)$ & OR [95\% CI, p-value] & Adjusted OR [95\% CI, p-value] \\
\hline 0 (non smoke) & $5(16.13)$ & $11(35.48)$ & 1 & 1 \\
$>0$ (smoke) & $26(83.87)$ & $20(64.52)$ & $2.86[0.86-9.56,0.088]$ & $3.46[0.96-12.55,0.058]$ \\
$<10$ & $5(16.13)$ & $14(45.16)$ & 1 & 1 \\
$>10$ & $26(83.87)$ & $17(54.84)$ & $4.28[1.30-14.08,0.017]$ & $4.88[1.41-16.87,0.012]$ \\
$<35$ & $13(41.94)$ & $24(77.42)$ & 1 & 1 \\
$>35$ & $18(58.06)$ & $7(22.58)$ & $4.75[1.57-14.31,0.006]$ & $4.94[1.62-15.12,0.005]$ \\
$<40$ & $19(61.29)$ & $25(80.65)$ & & 1 \\
$>40$ & $12(38.71)$ & $6(19.35)$ & $2.63[0.84-8.29,0.098]$ & $2.77[0.87-8.86,0.086]$ \\
\hline
\end{tabular}

adjusted multiple logistic regression for GSTM1 polymorphism 
GSTM1 Null Polymorphism and History of Tobacco Smoking among Oral Cancer Patients in Northeastern Thailand Table 4. Pack-years and Risk for Oral Cancer in Males

\begin{tabular}{lrrlc}
\hline Smoking pack-years & Cases n $(\%)$ & Controls n $(\%)$ & OR [95\% CI, p-value] & Adjusted OR ${ }^{a}$ [95\% CI, p-value] \\
\hline$\leq 5$ & $7(22.58)$ & $17(54.84)$ & 1 & 1 \\
$>5$ & $24(77.42)$ & $14(45.16)$ & $4.16[1.23-14.70,0.018]$ & $4.46[1.45-13.74,0.009]$ \\
$\leq 10$ & $12(38.71)$ & $22(70.97)$ & 1 & 1 \\
$>10$ & $19(61.29)$ & $9(29.03)$ & $3.87[1.19-12.84,0.021]$ & $3.89[1.34-11.28,0.012]$ \\
$\leq 15$ & $17(54.84)$ & $23(74.19)$ & 1 & 1 \\
$>15$ & $14(45.16)$ & $8(25.81)$ & $2.37[0.72-8.03,0.184]$ & $2.43[0.83-7.14,0.107]$ \\
\hline
\end{tabular}

adjusted multiple logistic regression for GSTM1 polymorphism

distribution between controls $v s$ cases of homozygous present (+/+), heterozygous present (+/-) and the null (-/-) genotype was $8.9 \%, 26.6 \%$ and $64.6 \%$ vs $7.6 \%, 38.0 \%$ and $54.4 \%$. The distribution of the GSTM1 genotypes was not significantly different between the cases and controls ( $>0.05$ ), and thus the GSTM1 null allele was not having an increased risk of oral cancer $v s$ the wild type (Table1).

When the focus was on the GSTM1 genotypes in nonsmoking females, neither the null genotype of GSTMI nor the heterozygous genotype altered the risk for OSCC $(p>0.05)$ (Table 2). The aspects of tobacco use were evaluated in the males; including duration and amount used (pack-years) (Tables $3 \& 4$ ). Subjects who smoked $\geq 10$ and $\geq 35$ years were at significant increased risks for OSCC with OR of 4.28 [95\%CI; 1.30-14.08, p=0.017] and 4.75 [95\%CI; 1.57-14.31, $\mathrm{p}=0.006]$ and adjusted OR of 4.88 [95\%CI; $1.41-16.87, \mathrm{p}=0.012]$ and 4.94 [95\%CI; $1.62-15.12, \mathrm{p}=0.005]$. A higher risk for OSCC was found for pack-years; those who smoked $>5$ and $>10$ pack-years had a respective OR of 4.16 [95\%CI; 1.23-14.70, $\mathrm{p}=0.018]$ and 3.87 [95\% CI; 1.19-12.84, $\mathrm{p}=0.021]$ and a respective adjusted OR of 4.46 [95\%CI; 1.45-13.74, $\mathrm{p}=0.009]$ and 3.89 [95\%CI; 1.34-11.28, $\mathrm{p}=0.012]$.

\section{Discussion}

GSTM1 polymorphism is one of the most studied loci vis-à-vis the risk of oral cancer. Homozygous deletion results in functional loss of GSTM1 enzyme (Gronau et al., 2003), which has been implicated in the genesis of several types of cancer (Schnakenberg et al., 2000; Tiwawech et al., 2005; Liu et al., 2014; Zakiullah et al., 2015). However, in this well designed case-control study with age - and sex-matched controls, the GSTM1 null genotype or the genotype distribution was not significantly associated with an increased risk of OSCC among northeastern Thais. This result confirmed no risk of association with the GSTM1 null among Caucasians (Deakin et al., 1996; Jourenkova-Mironova et al.,1999; Hahn et al., 2002) and Japanese (Tanimoto et al., 1999). As shown in a previous meta-analysis, which revealed no increased oral cancer risk among Chinese subjects carrying the GSTM1 null $(\mathrm{OR}=1.41,95 \% \mathrm{CI}: 0.72-2.77, \mathrm{p}=0.31)$ in contrast to a significant association between the GSTM1 genotype and oral cancer among Indians (OR=1.59, 95\% CI: 1.20-2.11, $\mathrm{p}=0.001$ ) (Peng et al., 2014), risk of oral cancer was not consistent with respect to the GSTM1 null. Inconsistency in the risk of GSTM1 null for oral cancer was extracted from many references showing for example, 1) the deletion of GSTM1 increased the risk for oral cancer (Sato et al.,
2000; Drummond et al., 2004), or 2) GSTM1 null gene polymorphism might result in an increased risk of oral cancer in Asians but not Caucasians (Zhao et al., 2014). We thus emphasize that the GSTM1 null polymorphism may be important in the ethnic specific risk of oral cancer. Ethnic and regional differences accord with lifestyle, which may affect the outcome of some genetic variations of the GSTM1 polymorphism. The diversity of association between the GSTM1 null genotype and oral cancer may differ by geographical region and socio-economic status. These assumptions are supported by the latitudinal cline in the distribution of the GSTM1 null genotype that is supposed to be the result of gene-environment adaptations (Saitou and Ishida, 2015).

Tobacco smoke is well documented as a crucial cause of various types of cancer (Sobue et al., 2002; Samanic et al., 2006; Petti, 2009; Loyha et al., 2012; Natphopsuk et al., 2015). In this study, of the GSTM1 genotype, interaction with smoking habit was not associated with risk of the OSCC. A similar finding vis-à-vis the GSTM1 genotype had no influence on oral cancer in non-smokers and occasional smokers but resulted in a higher risk in frequent smokers in Spain (Varela-Lema et al., 2008). While a meta-analysis, however, revealed an association between the GSTM1 null genotype and a higher risk of oral cancer among Asians but not Caucasians; GSTM1 polymorphism might modify the relation between smoking status and oral cancer risk (Zhang et al., 2011). It implies that smoking is more detrimental to persons who carry the GSTM1 gene in homozygous or heterozygous status. Our results showed that those who had smoking habit for $>10$ or $>35$ years but not more than 40 years were at high risks (Table 3 ). This trend, much longer smoking history reduces the risk, was previously also reported; among those who have survived against much longer and/or larger exposure to smoking, the risk of smoking seems to be masked and other critical risk(s) than smoking may become tangible. We also found the higher risks of smoking pack-years for $>5$ and $>10$; this suggests that at high amounts and longer durations of smoking there is an accumulation of carcinogens, which overwhelm detoxification systems, leading to carcinogenesis. When the ORs were calculated only among smokers, none of the categories showed significant (data not shown). This indicates that the status of smoking or non-smoking is highly critical in the development of oral cancer. In conclusion, these findings suggest certain smoking patterns are at greater risk and to reduce the risk of smoking-related cancer, both smoking duration and pack-years should be addressed. 


\section{Acknowledgements}

This work was supported in part by (a) an Invitation Research Grant from the Faculty of Medicine, Khon Kaen University, (b) a Research Fund from the Khon Kaen University (c) a Grant from Chulabhorn International College of Medicine Research Fund 2015 (d) JSPS Core University Program. The authors thank the doctors, nurses and volunteers who supported this study and Mr. Bryan Roderick Hamman for assistance with the Englishlanguage presentation of the manuscript.

\section{References}

Deakin M, Elder J, Hendrickse C, et al (1996). Glutathione S-transferase GSTT1 genotypes and susceptibility to cancer: studies of interactions with GSTM1 in lung, oral, gastric and colorectal cancers. Carcinogenesis, 17, 881-4

Drummond SN, De Marco L, Noronha JC, Gomez RS. (2004) GSTM1 polymorphism and oral squamous cell carcinoma. Oral Oncol, 40, 52-5.

Gronau S, Koenig-Greger D, Jerg M, Riechelmann H (2003). GSTM1 enzyme concentration and enzyme activity in correlation to the genotype of detoxification enzymes in squamous cell carcinoma of the oral cavity. Oral Dis, 9, 62-7.

Hahn M, Hagedorn G, Kuhlisch E, Schackert HK, Eckelt U (2002). Genetic polymorphisms of drug-metabolizing enzymes and susceptibility to oral cavity cancer. Oral Oncol, 38, 486-90.

Hayes JD, Pulford DJ (1995). The glutathione S-transferase supergene family: regulation of GST and the contribution of the isoenzymes to cancer chemoprotection and drug resistance. Crit Rev Biochem Mol Biol, 30, 445-600.

Jourenkova-Mironova N, Voho A, Bouchardy C, et al (1999). Glutathione S-transferase GSTM1, GSTM3, GSTP1 and GSTT1 genotypes and the risk of smoking-related oral and pharyngeal cancers. Int J Cancer, 81, 44-8.

Larizadeh MH, Shabani M (2012). Survival following non surgical treatments for oral cancer: a single institutional result. Asian Pac J Cancer Prev, 13, 4133-6.

Liu X, Li Z, Zhang Z, et al (2014). Meta-analysis of GSTM1 null genotype and lung cancer risk in Asians. Med Sci Monit, 20, 1239-45.

Loyha K, Vatanasapt P, Promthet S, Parkin DM (2012). Risk factors for oral cancer in northeast Thailand. Asian Pac J Cancer Prev, 13, 5087-90.

Mukherjee JJ, Kumar S (2010). Phenolic fraction of tobacco smoke condensate potentiates benzo[a]pyerene diol epoxideinduced cell transformation: role of protein kinase C. Mutat Res, 696, 89-94.

Nagler R, Ben-Izhak O, Savulescu D, et al (2010). Oral cancer, cigarette smoke and mitochondrial $18 \mathrm{kDa}$ translocator protein (TSPO) - In vitro, in vivo, salivary analysis. Biochim Biophys Acta, 1802, 454-61.

Nair UJ, Nair J, Mathew B, Bartsch H (1999). Glutathione S-transferase M1 and T1 null genotypes as risk factors for oral leukoplakia in ethnic Indian betel quid/tobacco chewers. Carcinogenesis, 20, 743-8.

Natphopsuk S, Settheetham-Ishida W, Settheetham D, Ishida T (2015). Lack of Participation of the GSTM1 polymorphism in cervical cancer development in northeast Thailand. Asian Pac J Cancer Prev, 16, 1935-7.

Peng J, Liu HZ, Zhu YJ (2014). Null Glutathione S-transferase $\mathrm{T} 1$ and M1 genotypes and oral cancer susceptibility in China and India--a meta-analysis. Asian Pac J Cancer Prev, 15, 287-90
Petti S (2009). Lifestyle risk factors for oral cancer. Oral Oncol, 45, 340-50.

Rodriguez T, Altieri A, Chatenoud L, et al (2004). Risk factors for oral and pharyngeal cancer in young adults. Oral Oncol, 40, 207-13.

Rogers SN, Brown JS, Woolgar JA, et al (2009). Survival following primary surgery for oral cancer. Oral Oncol, 45, 201-11.

Roodi N, Dupont WD, Moore JH, Parl FF (2004). Association of homozygous wild-type glutathione S-transferase M1 genotype with increased breast cancer risk. Cancer Res, 64, 1233-6.

Saitou M, Ishida T (2015). Distributions of the GSTM1 and GSTT1 null genotypes worldwide are characterized by latitudinal clines. Asian Pac J Cancer Prev, 16, 355-61.

Samanic C, Kogevinas M, Dosemeci M, et al (2006). Smoking and bladder cancer in Spain: effects of tobacco type, timing, environmental tobacco smoke, and gender. Cancer Epidemiol Biomarkers Prev, 15, 1348-54.

Sato M, Sato T, Izumo T, Amagasa T (2000). Genetically high susceptibility to oral squamous cell carcinoma in terms of combined genotyping of CYP1A1 and GSTM1 genes. Oral Oncol, 36, 267-71.

Schnakenberg E, Breuer R, Werdin R, Dreikorn K, Schloot W (2000). Susceptibility genes: GSTM1 and GSTM3 as genetic risk factors in bladder cancer. Cytogenet Cell Genet, 91, 234-8.

Shields PG (2002). Molecular epidemiology of smoking and lung cancer. Oncogene, 21, 6870-6.

Sobue T, Yamamoto S, Hara M, et al (2002). Cigarette smoking and subsequent risk of lung cancer by histologic type in middle-aged Japanese men and women: the JPHC study. Int J Cancer, 99, 245-51.

Sreelekha TT, Ramadas K, Pandey M, et al (2001). Genetic polymorphism of CYP1A1, GSTM1 and GSTT1 genes in Indian oral cancer. Oral Oncol, 37, 593-8.

Tanimoto K, Hayashi S, Yoshiga K, Ichikawa T (1999). Polymorphisms of the CYP1A1 and GSTM1 gene involved in oral squamous cell carcinoma in association with a cigarette dose. Oral Oncol, 35, 191-6.

Tiwawech D, Srivatanakul P, Karalak A, Ishida T (2005). Glutathione S-transferase M1 gene polymorphism in Thai nasopharyngeal carcinoma. Asian Pac J Cancer Prev, 6, 270-5.

Varela-Lema L, Ruano-Ravina A, Juiz Crespo MA, et al (2008). CYP1A1, mEH, and GSTM1 polymophisms and risk of oral and pharyngeal cancer: a spanish case-control study. J Oncol, 741310.

Vatanasapt P, Suwanrungruang K, Kamsa-Ard S, et al (2011). Epidemiology of oral and pharyngeal cancers in Khon Kaen, Thailand: a high incidence in females. Asian Pac J Cancer Prev, 12, 2505-8.

Zakiullah, Ahmadullah, Khisroon M, et al (2015). Genetic susceptibility to oral cancer due to combined effects of GSTT1, GSTM1 and CYP1A1 gene variants in tobacco addicted patients of pashtun ethnicity of khyber pakhtunkhwa province of Pakistan. Asian Pac J Cancer Prev, 16, 1145-50.

Zhang ZJ, Hao K, Shi R, et al (2011). Glutathione S-transferase M1 (GSTM1) and glutathione S-transferase T1 (GSTT1) null polymorphisms, smoking, and their interaction in oral cancer: a HuGE review and meta-analysis. Am J Epidemiol, 173, 847-57

Zhao SF, Yang XD, Lu MX, et al (2014). GSTM1 null polymorphisms and oral cancer risk: a meta-analysis. Tumour Biol, 35, 287-93. 